\title{
The Aesthetics of Transformation
}

\author{
By Vladimir Mako*
}

In the context of the traditional definition of aesthetics as the "science on beauty", the title of this paper can be understood as a contradiction in the first place. Traditional search for beauty is the search for completeness, balance and wholeness. However, the contemporary theories on aesthetics, particularly in architecture and urban design, insists on the dynamism of perception which is based among other principles, on aspects of ambiguity, ambivalence, actually on effects of transformation and the sense of perceivers aesthetic participation. These aspects are tools of creative thinking, and usually they can be defined in a different way than in traditional theories. That is the reason why the aesthetics of transformation exists preferably as an urban concept, referring on the sensation of constant dynamic changes of the build environment.

\section{Introduction}

The aim of this paper is to discuss a particular issue that appears in the context of contemporary aesthetics of architecture and urban design, regarding the notion of transformation. At the beginning, we should differentiate two positions that this notion is establishing within the aesthetic discipline. The first one is related to the expression of "the transformation of aesthetics". It indicates a historical process of changes by which aesthetics developed philosophically and theoretically, reaching different understandings of social and cultural influences on its system of perception and judgment of values in a particular period of time. It consists on a slow, rational and analytical approach to this investigation, and it will not be in the focus of our discussion.

We will concentrate on the second expression using the notion in focus, "the aesthetics of transformation". In this theoretical construct the notion of transformation is indicated as the very essence of the aesthetic process. By this, one is evaluating the process of transformation itself as a fluid flow of constant reevaluation of aesthetic values, based on the observer's point of perception. This is a phenomenon that some scholars are positioning in the core of the effect that an object provokes within the emotional aesthetic experience of the observer. ${ }^{1}$ According to the nature of the emotional aesthetic experience, one can say that, as a subjective value, it is in its essence transformable. In this sense, "the aesthetics of transformation" is establishing a process which engages directly to the fundaments of the observer's perception', its reflection on aesthetic values, and it presents itself as a constant search for new social and cultural positions, a permanent change of an aesthetic focal point.

* Professor, University of Belgrade, Serbia.

1. C. Bell, "The Aesthetic Hypothesis," in Aesthetics, ed. Susan Feagin and Patrick Maynard (Oxford: Oxford University Press, 1997), 16. 
The inspiration for establishing this theoretical position came from the Nouvel's notion of "the aesthetics of disappearance", as the prelude for the "metamorphosis of architecture". If something disappears, something else appears, and usually we can imagine it as a completely different perceptual value and meaning. Here, disappearance can be thought as a mental dynamic reflection preconditioning the "aesthetics of transformation", and appearance of a completely different aesthetic sensation. This position is possible because it is based and supported by our intuitional capacity to experience and evaluate diversity of sensorial effects almost immediately we see it, even if they are completely different. It is a reaction at the "first glance" perception, which operates without consciously defined rational and analytical mode, but rather it grasps its power from the intuitional domain of human knowledge, and its experiential capacity.

One of the scholars indicating the importance of this capacity, Dewey, links it to the character of the actual world. ${ }^{3}$ Its dynamic manifestation through which the people are moving from disturbance to harmony, and vice versa, in their perception and by that in their experience of the world, has a particular value. It is the catalyst of their intuitional power to manage the phenomenon of persistent transformation in their life, and according to that in the build environment, as a part of it.

For better understanding of this issue, we should recall on Gadamer's understanding that transformation is essentially different from alteration, because it does not employ the notion of maintaining the similarity with the previous perceptual value. ${ }^{4}$ By transformation, a perceptual value is reaching an absolute autonomy in the sense of aesthetic properties, as in the way of how we perceive it. However, Gadamer established its position regarding the art of theater, and in that context we can understand the possibility of such a concept of transformation. But, in architecture and urban design, as artistic expressions consisting on mainly permanent material and sensorial values, there is always a question hanging over our heads, as the Theocle's sward, and it can be compressed in one single expression: what is transforming into what? How a value established by material means can be transformed into completely different sensorial experience, avoiding alteration in this process?

It seems that there are more similarities between Gadamer's theatrical examples and architecture and urban design, than one can think. We should remember that space also occupies time. The process of transformation how it was explained by Gadamer, also employs space and time. Transformation relies on the perceiver's position in time by which he perceives the value materialized in space. There is always a double acting in such a process: the actor is transforming his personality into new existing difference, and the observer confirms this new value by its emotional reaction. It proves that the virtual, as a mode of existence is equally aesthetically valuable as that what we

2. J. Baudrillard and J. Nouvel, The Singular Objects of Architecture (University of Minnesota Press, 2002), 17-18.

3. J. Dewey, "The Aesthetic in Experience," in Aesthetics. 1997, op.cit. 46.

4. H-G Gadamer, Truth and Method (London: Continuum, 2004), 110-111. 
usually call real. What one see, that exists, particularly if it is emotionally responded.

In the case of architecture and urban design, the observer probably plays a more important role than in other arts, when it comes to the aesthetic sensation of transformation. Here, perception is pregnant with sensorial effects existing in a dynamic process of engagement with an object or city site. It can be directed by the designer or by life processes and needs, but it is usually a potential for transformation of perceptual values. This notion is very close to the Kwinter's idea of the "space as an event that is always in the process of becoming". It means that aesthetics of transformation in architecture employs activity and openness for different external influences, but which the final result is far from the notion of atmosphere. In this context, transformation as an aesthetic value cannot be thought as a result of changing parameters of a form, added or taken off. It is an open condition, a process where needs of life are accepted as the driving force of transformation. It employs the distance and the intuitive social capacity of the observer/participator with the object or site that transforms. That is also the reason why subjectivity of the observer/actor cannot be implemented in the process, as Gadamer emphasized. As the scene transforms perceptually, and by this socially and culturally, so our own transcultural being annuls our rational component of aesthetic perception.

Transformation as a core of aesthetic sensation seems to emerge inbetween two particular poles: perception as an active element and as Grosz emphasized, by that "related primarily to an impending future"; 6 and memory as inactive and virtual force. However, memory has its own value in the process, particularly when it activates its sense for transcultural aesthetic communication. By that, a new perceptual value, reached by transformation, can be socially and culturally processed by our intuition and finally aesthetically accepted as a variable experience and value. This new intuitive knowledge is the fundament for future creativity, which attempts to coordinate the new logic of invention through means as "ingenuity, experimentation, novelty, specification, and particularity, and by that it focuses on an intuition of uniqueness."7 It seems that these means can be even thought as aesthetic guide points for the process of transformation itself, by which it actualizes as a mental transformation of the perceiver, challenging the standard cultural meanings expressed through architecture and a build environment.

In order to understand this issue better, we will analyze four examples of architectural and environmental perceptual values that can indicate aesthetic variables reached by the process of transformation. Although the process of transformation as an aesthetic quality should be understood as a broader phenomenon, through these four examples we will be able, for the purpose of our discussion, to explain the essential idea regarding the topic in focus. These

5. S. Kwinter, Architectures of Time (Cambridge Mass: The MIT Press, 2002).

6. E. Grosz, Architecture from the Outside: Essays on Virtual and Real Space (The MIT Press. 2001), 119.

7. Ibid, 125. 
examples will not be analyzed as generalized values, but they will expose possibilities in reaching particularities trough the process that we discuss.

The first example CaixaForum, finished in Madrid in 2008 by Herzog and De Meuron, indicates the sense of disappearance of perceptual continuity as one of the notions of transformation in aesthetic value in architecture (Figures 1 and 2). It seems that designers challenged the steps in the line of expectations developed by the usual educational system in architecture, which understands that the logic of the interior of a building should be altered to the perceptual values of the exterior in its sense of dynamism, cultural acceptance, and functional disposition, and even materiality. Actually, what we perceive inside the building transforms the primal contact with the outside values of the object into a completely different aesthetic position.

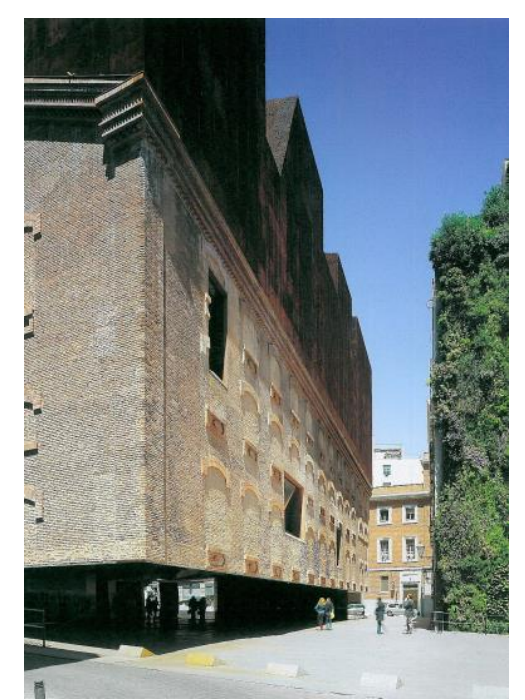

Figure 1. CaixaForum, Herzog \& De Meuron Source: Author.

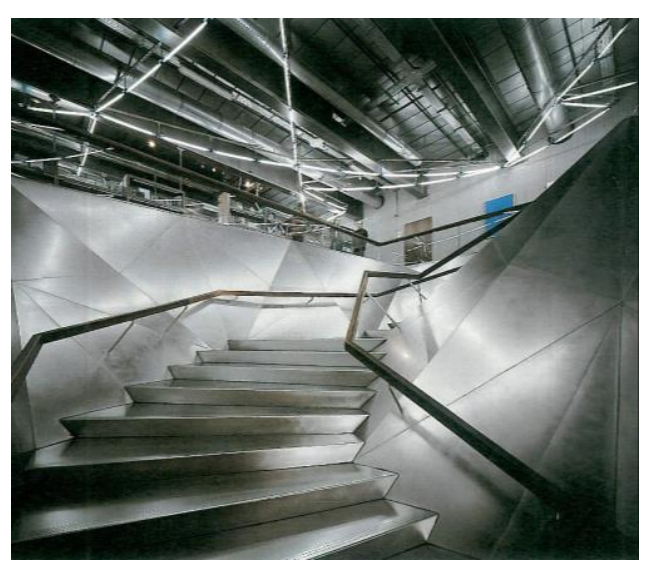

Figure 2. CaixaForum, Herzog \&De Meuron Source: Author.

Metal versus brick, cubo-futuristic particles versus plane surface, dynamism of light and reflections versus monotonic perceptual firmness of the form, hi-tech interior structure versus traditional cubic form, are just some of 
the characteristics that bring the transformation of perceptual experience into the creative core of this building. Even the effect of the levitation of the external building surfaces indicates the existence of aesthetic contra standard after we enter the architecture. However, our transcultural memory, the intuitional knowledge that we developed, is maintaining the particularity of this creative logic. One can sense the presence of the feeling of primordial structures, often used in early avant-garde architecture, ${ }^{8}$ which, after the first violent sensation, tries to establish the equilibrium of presented contra standard aesthetic elements. Actually, in a stone or ground, possibly identified in the brick façade and cubic form (Figure 1), one can expect to found metal or crystalline structure (Figure 2). However, even if intuitionally recognized, the whole effect brings up a new aesthetic value, trough the dynamism of confrontation, and transformation of perceptual categories.

In this context, the materials confronting in the process of perception are building up a sense of different realities, while according to Herzog's words the reality finds its manifestation in materials it is build from. ${ }^{9}$ However, intuitively belonging to both of these realities, we are able to contextualize them as one in our mind.

This example brings up a few other important aspects of the creative approach to architecture. As Hays emphasized in his reflection on Boudrillard and Nouvel's book of interviews, there is a great importance in invention of new techniques for rethinking issues of representation, ${ }^{10}$ which concerns the permanent ties between architecture and new aesthetic, even philosophical ideas. Similar to Boudrillard's idea of a constructed object which enables experience of instability of space, ${ }^{11}$ here we have a translation of an intuitional primordial sense into a contemporary dynamic technological structure, integrating surface, light, materiality, and subconscious sensitivity into an unbreakable perceptual whole.

The next example, the Zara building in Tokyo, again build by Herzog and DeMeuron, refers on the transformation of perceptual values as a result of changing distances between the observer and the object. The dynamism of the observer's movement towards the building transforms the perceptual value of the form and the external surface, consisting on the structure of glass prisms and the reflection of the environment on it (Figure 3), into a number of particles framing the images of the interior which starts to reveal itself (Figure 4). It seems that in the works of Herzog and De Meurone the concept of façade takes a particular form of expression. ${ }^{12}$ It is conceptualized to merge images, questioning the continuous flow of sensations and the perceiver's sense of

8. V. Mako, "Aesthetic Sensibility of Primordial Structures in the 20th Century Architecture," in Proceedings of the III Mediterranean Congress of Aesthetics. Koper. 2006 (Ljubljana: Slovenian Society for Aesthetics, 2007), 104-107.

9. P. Ursprung, "Introduction: Exibiting Herzog and De Meuron," in Herzog \& De Meuron Natural History, ed. Philip Ursprung (Montreal: Canadian Centre for Architecture and Lars Muller Publishers, 2005), 31.

10. M. Hays, "Introduction," in Baudrillard and Nouvel, op. cit. x.

11. Boudrillard and Nouvel, op.cit. 4.

12. Ursprung, op.cit. 31. 
scale, when experiencing the fragments of interior exposed by transparency of the envelope.

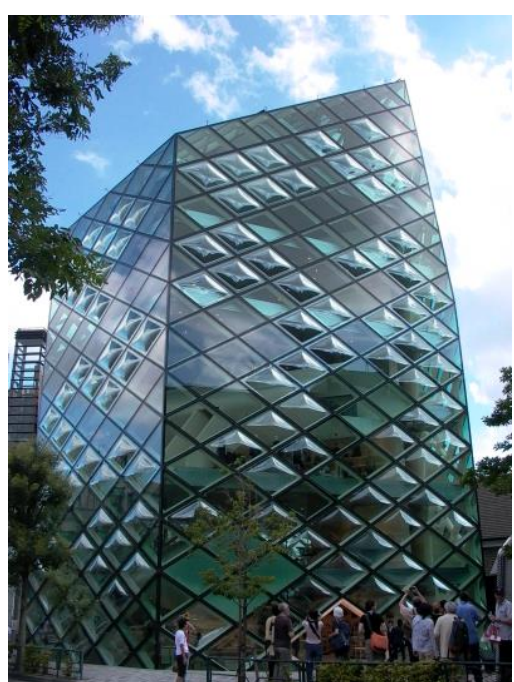

Figure 3. Herzog and De Meuron Zara, Tokyo

Source: Author.

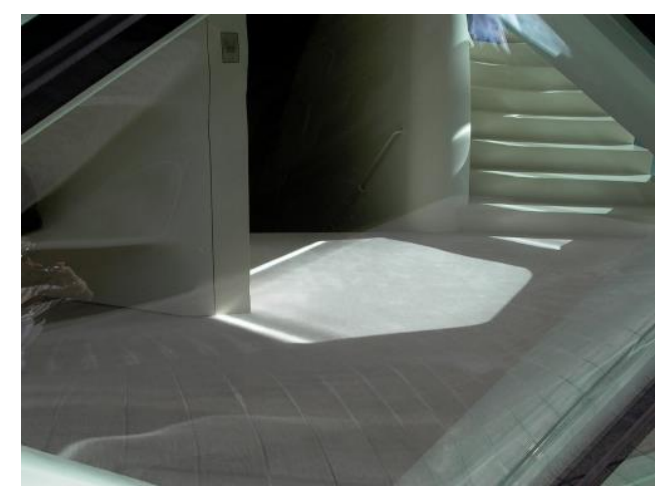

Figure 4. Herzog and De Meuron Zara, Tokyo, View of a Particle Source: Author.

The process of this transformation happens before the observer's eyes, changing the reflecting façade into transparent surface consisting on interior particles, forming a structure of incredible ambiguity. The sense of existing interior structure complements the feeling of the mirroring nature of the facade, by which the image of the interior particle reflects on the external surface. The look into the depth of the space is through a screen like mirroring surface, where components of real structure and materiality do not exist anymore. In this context, light remains the only element of structural firmness of the inner space which becomes an image of virtual power. It is one of the processes transforming perception, where the observer's experience is directly confronted 
with the character of the building. It is one-to-one experience as Herzog explained. $^{13}$

Through the observer's movement and the dynamism of sensorial transformation, the surface of the building becomes an event, which engages our mental capacity to accept the image of the transformed pictorial value. Through disappearance of the surface reflecting external environmental sensorial values, appears a surface which reflects on itself the parts of interior structure characterized by light and shadow (Figure 4). Trough the surface of the building the exterior world and the interior structure communicate, and they are mentally connected in the observer's mind. They achieve different levels of sensorial activities regulated by the observer's movement and the distance from the objects surface. However, by this transforming activity, the object itself disappears. The opposed nature of two reflections, building the sense of ambiguity of perceptual values, transforms the surface of the architecture into the real spatial dimension. It becomes the primal holder of the visual character and the aesthetic value of the architectural object. The explained perceptual sensations reflect on Herzog and De Meuron's ideas regarding the transformation of the physical world into a spiritual concept. Actually, they establish a conceptual level of perception in architecture. ${ }^{14}$

The third example is in a way similar to the previous one just discussed. The design of both buildings emphasizes the notion of surface as the primal actor in communication of transforming aesthetic activity. However, the surface of the Dior building in Tokyo, designed by Sejima, develops a perceptual quality which captures the sense of translucency of a screen, at a particular daily moment (Figure 5).

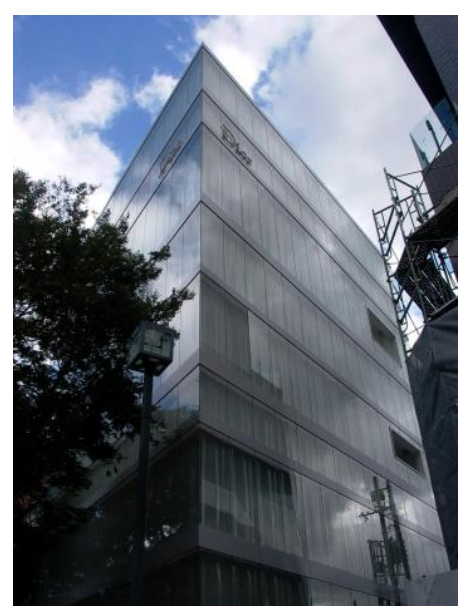

Figure 5. Sejima, Dior, Tokyo, General View Source: Author.

13. Interview conducted in Basel in 2002 by Ursprung, in Herzog \& De Meurone, op.cit. 81-82.

14. W. Wong, Herzog \& de Meuron (Basel: Birkhauser Verlag. 1998), 185-186. 


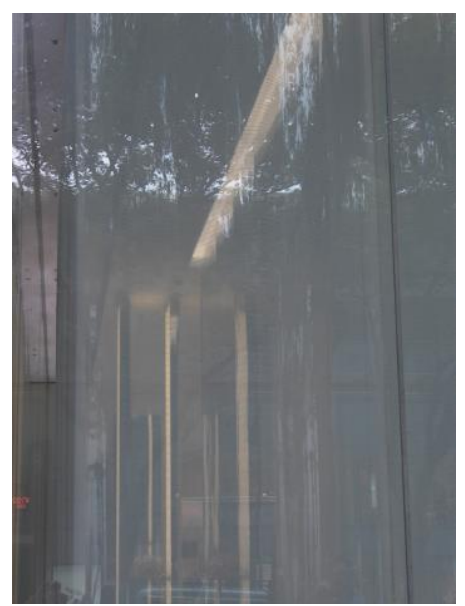

Figure 6. Sejima, Dior, Tokyo, Screen Space Source: Author.

The effect that can be captured is based on a sense of the feeling of virtual, high technological and dematerialized space. It is close to the Baudrillard's notion of "space of the screen, mental space"15 by which we more feel what should be revealed than actually seeing what is exposed behind the screen. Essentially, this activity transforms an opaque surface into a translucent screen of vague spatial depth. Actually, one cannot see the space but its indication formed intuitively by the feeling of the flow of light that captures the undefined objects existing in that what we sense as space (Figure 6).

Theoreticians as Grosz, these effects explain trough the influence of the concept of cyber space, which involves into architecture "a disembodied, nonmaterial, or transcendental notion of design, design disembodied from matter." ${ }^{16}$ However, in this example we are not talking about a finalized design sensation, but emphasizing its constant dynamic transformable activity. The named conceptual characteristics we can accept as general aesthetic values, but their active interaction always forms, at least a slightly different effect in a particular moment. At the same time, perception of this phenomenon structuralizes mental capacity of the observer to participate in the process of "revelation" of that what is behind the screen. It is a form of inventive perception, a full intuitional capacity of active aesthetic appreciation of architecture.

However, exposing as virtual trough its general characteristics, the whole sensation of the space of the screen reflects the existence of a real world behind the surface. Probably activated by the nature of intuitional knowledge, which is basically empirical, our perception of the transformation of the surface into the screen space, guides our imagination towards a construct of real. It can be thought as a pure longing, while the perceiver will never observe the exactness of the space behind the screen.

The aesthetic effects of transformation are also visible in examples concerning particular aspects of urban design. Maybe it will be more correct if

15. Boudrillard and Nouvel, op.cit. 10.

16. Grosz, op.cit. 85 . 
instead of the term urban we use the notion cultural build environment. By this we emphasize the nature of the complex structure that culture and build environment form, particularly when it comes to the activities leading towards the aesthetic of transformation.

The contemporary notion of build environments is extremely complex. For this reason, our discussion should pay attention on a few aspects, important for our elemental understanding what transformation in build environment can be. The role of architecture, commerce, transcultural nature of people's communication, and dynamic sensorial nature of perception, are components through which we will analyze some of the important aspects of aesthetics of transformation concerning the build environment. Our interest in this topic narrows our focus on those areas of urban agglomerations, where the named components are in fundamental interaction, producing new aesthetic activity and values.

It is obvious that perception of a build environment engage all senses in a very dynamic way. As Susan Sontag emphasized, the contemporary experience of urban (build) environment, is based on massive cultural overproduction, where the recognition of exact characters and meanings of the sensorial sensations are not recognizable any more. ${ }^{17}$ This is particularly evident in the cases where the observer does not belong to the culture which developed the structure that is experienced. In this context, we will take an, more or less, radical example. The position of an ordinary European visitor of Tokyo can be in this context very useful. Despite the possibility to read only a few inscriptions, mostly advertising or brand, the visitor is literary blind (Figure 7). Its communication with a number of important functions of the city is interrupted, especially if we are keeping in mind that a city is not only a matrix of urban and architectural forms, but in a large part a structure of different functions, advertising, and social contents. All these aspects could be thought as important for a social identification in a city, forming a visual culture which provides elements for aesthetics of social behavior, ${ }^{18}$ which reflects also on a temporal visitor of a city. Visitors, not being able to communicate trough inscriptions, can not indicate the functions in a complete way. Therefore he/she cannot fulfill their interrelationship of functions as the essential value of the perception of the build environment as the human environment. The visitor is, in this context, directed to the images, and to the process of recognizing the functions according to its own European experience.

17. S. Sontag, “Against Interpretation.” in Aesthetics (1997) op.cit. 255.

18. A. Madanipour, "Social Exclusion and Space," in The City Reader (New York: Routledge, 2005), 183-184. 


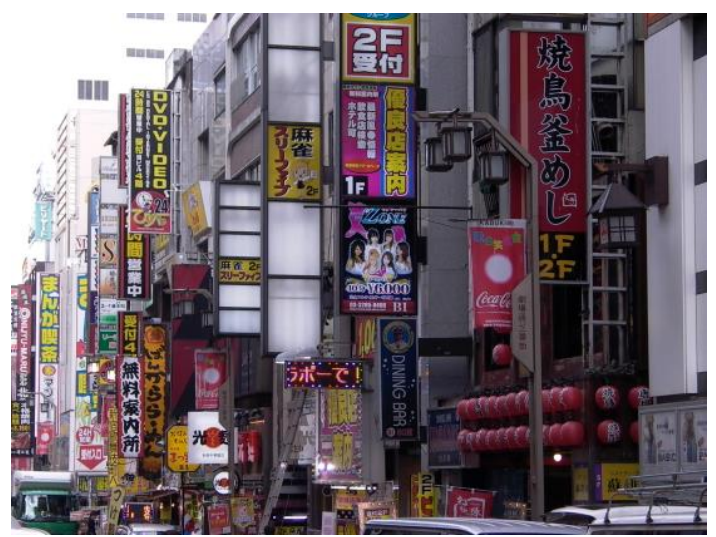

Figures 7. Tokyo, Street Commercials

Source: Author.

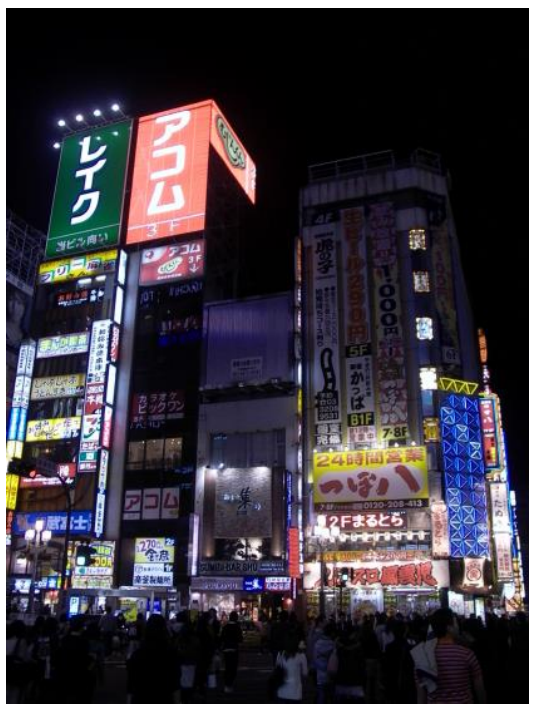

Figures 8. Tokyo, Street Commercials

Source: Author.

Here, we can indicate a cultural paradox. To start an aesthetic perception, the visitor's cultural being is processing elements recognizable according to its own cultural matrix. In this sense, one is relying on the forms of cultural identity that can be recognized, for, "at the same time quite different forms of identity can exist in the same place." ${ }^{19}$ Perceiving parts of the urban structure developed by modern and contemporary architectural ideas, the visitor is able to process only the architectural and urban forms, because they are based on the architectural tradition of the western culture. More than that, this tradition is mainly avoiding the exterior presentation of the character of the inner function. Without recognizable marks and inscriptions, the visitor's identification of the most of architectural functions and contents is very difficult. In that sense, to the European visitor of Tokyo, the most of the functional and social content of the perceived architecture still remains opaque,

19. W. Welsch, "Transculturality: The Changing Form of Cultures Today." in Filozofski vestnik 2/2001 (Ljubljana: Institute of Philosophy at ZRC SAZU, 2001), 62. 
which is causing the disruption in the usual way of perception of the urban totality, composed of forms, functions, and social activities. Ideograms, language, advertising and local brand names and inscriptions, marking functions and social contents, are remaining out of the visitor's scope. They become an abstract expression of graphic forms and colors. Therefore, the aesthetic experience mainly rests on the dynamic perception of abstract images, and it is directed almost towards pure artistic sensations (Figure 8).

However, the analyzed process shows that the experience of a build environment, containing diverse traditional and contemporary architectural forms, is a very complex one. It asks for a dynamic perceptual activity, and for engagement of the complete cultural background of the observer. It activates all of one's cultural sensibility, and the ability of recognition and evaluation of a range of sensorial values. In this context, the appearance of pure artistic sensations in perceiving an urban matrix, divorced from the real understanding of functional and social content, is an important issue. It leads us toward a particular appearance and experience of a virtual reality, which is the core of a few theories regarding the perception of urban and architectural structure. Mainly, virtual reality provides conditions for a total sensory experience of an urban structure, ${ }^{20}$ and theoretically, leads towards definition of the aesthetic of transformation regarding a build environment.

The virtual reality on the general level is not developed on the impression of the whole structure, but on the characteristics of a few perceived sites and fragments of a build environment, which actually become a cultural and aesthetic environment. That means that the nature of the virtual reality is a transformable value, according to the sensorial effects, and how one experiences them. Each new set of sensorial effects of different components of the environment, are producing new cultural experience, and therefore new sense of virtual reality. As defined by Baudrillard and Nouvel, the notion of the aesthetics of disappearance is close to particular sense of perceptual instability of space, even a concept of illusion, which is based on the fiction of a society. ${ }^{21}$ In a way, the named characteristics are reflecting on our previous discussion on perceptual values, and aesthetic experience of their transformation. We should not forget that the explained perceptional character is essentially an emphatic form of "inter subjective experience which becomes the condition for possible knowledge of the existing outher world", as Parviainen defined it. ${ }^{22}$

The notion of the concept of illusion can be thought more as the collective effect of other elements appearing in the urban structure, as commercials and advertising inscriptions and images, screens and different art installations, new concepts of materialization of architecture, than a rational assumption projected in space. All these elements are developing sensorial values of dematerialization of architecture, and they challenge our three dimensional

20. Y. Sepanmaa, "Multy - sensoriness and the City," in The Aesthetics of Human Environments (Broadview Press, 2007), 95.

21. Baudrillard and Nouvel, op.cit. 4,6.

22. J. Parviainen, "Kinaesthetic Empathy," in Dialogue and Universalism XIII, no. 11-12 (Polish Academy of Sciences and Warsaw University, 2003), 160. 
perception of it. It is a process of "blending the real image and the virtual image", as Nouvel defined it, and it leads to a new perceptual value: towards the virtual sense of space, "which is complicit with hyper reality." 23 The reflection on the mental space complementary to the space of the screen is a very important one, particularly in our case study where the visitor of Tokyo is especially oriented towards this kind of sensorial effects. As Baudrillard emphasized, this kind of "aestheticization is not part of the real, but they are becoming values, assume values," 24 according to personal experience of an urban and architectural structure. The whole process is actually based on the constant transformation of sensorial values, and we can indicate a few steps in this process: first, the perception of the existing formal structure of an urban matrix, architecture, and other effects linked to them; second, the establishment of the fiction of a society, in this case of a foreign origin, according to the perceived sensorial values; third, an effort of the perceiver to form a range of aesthetic values according to the established social fiction. In this context, it is important to note that all indicated steps are mainly guided by a personal cultural presumption of the observer, related to the sensorial effects of the build environment, and the way one perceives its functional and social content. However, as Welsch emphasized, the process of transcultural evaluation is not a simple uniformization. It is the production of a new diversity. ${ }^{25}$ It seems that the precondition of the possibility to perceive in this way is based on new digital and electronic culture we belong in general, where the perceiver "reacts on the immediate effects of intensity and the aspects of time and structures, as the consequence of use of samples without their meaning."26

All presented examples indicate that the nature of the aesthetics of transformation springs out from the immediate intuitive experience of new sensorial values, resulting from changing distances, Medias and models of expression, technology, and the transcultural focal point of the observer. Transformation is related to the sensitivity of the perceiver, and its ability to aesthetically indicate appearance and disappearance of potentials cultural and sensorial, that are forming values in an aesthetic experience. Is the aesthetic of transformation a projected phantasm, a result of imagination, a creativity transforming "now" into "future"? Whatever the answer would be, it is based on that what we see, and not on what we comprehend. The nature of aesthetics of transformation can be imagined to be a constant growing effect, a process as an open spiral, integrating creative efforts and human needs, vital living and cultural conditions.

23. Baudrillard, and Nouvel, op.cit. 8.

24. Ibid, 21.

25. Welsh, op.cit. 80 .

26. W. Jauk, "The Transgression of the Mechanistic Paradigm - Music and the New Arts," in Dialog and Universalism (2003) op.cit. 179. 
Bibliography

Baudrillard, J., and Nouvel, J. 2002. The Singular Objects of Architecture. University of Minnesota Press.

Bell, C. 1997. The Aesthetic Hypothesis. In Aesthetics. Ed. Susan Feagin and Patrick Maynard. Oxford University Press. Oxford.

Dewey, J. "The Aesthetic in Experience." In Aesthetics. Edited by Susan Feagin and Patrick Maynard. Oxford: Oxford University Press, 1997.

Gadamer, H.-G. Truth and Method. Continuum. London: 2004.

Grosz, E. Architecture from the Outside: Essays on Virtual and Real Space. Massachusetts Institute of Technology: The MIT Press, 2001.

Hays, M. "Introduction." In The Singular Objects of Architecture. Edited by Baudrillard and Nouvel. 2002.

Jauk, W. "The Transgression of the Mechanistic Paradigm-Music and the New Arts." In Dialogue and Universalism XIII, no. 11-12. Polish Academy of Sciences and Warsaw University, 2003.

Kwinter, S. Architectures of Time: Toward a Theory of the Evant in Modernist Culture. Cambridge Massachusetts: The MIT Press, 2002.

Madanipour, A. "Social Exclusion and Space." In The City Reader. New York: Routledge, 2005.

Mako, V. "Aesthetic Sensibility of the Primordial Structures in the $20^{\text {th }}$ Century Architecture." In Proceedings of the III Mediterranean Congress of Aesthetics. Koper. 2006. Ljubljana: Slovenian Society of Aesthetics, 2007.

Parviainen, J. Kinaesthetic Empathy. In Dialogue and Universalism XIII, no. 11-12. Polish Academy of Sciences and Warsaw University, 2003.

Sepanmaa, Y. "Multy - Sensoriness and the City." In The Aesthetics of Human Environments. Broadview Press, 2007.

Sontag, S. "Against Interpretation.” In Aesthetics. Edited by Susan Feagin and Patrick Maynard. Oxford: Oxford University Press, 1997.

Ursprung, P. "Introduction: Exhibiting Herzog and De Meuron.” In Herzog and De Meuron Natural History. Edited by Philip Ursprung. Montreal: Canadian Centre for Architecture and Lars Muller Publishers, 2005.

Welsch, W. "Transculturality: The Changing Form of Cultures Today." In Filozofski Vestnik 2/2001. Ljubljana: Institute of Philosophy at ZRC SAZU, 2001.

Wong, W. Herzog \& de Meuron. Basel: Birkhauser Verlag, 1998. 
